\title{
Potential risks of spectrum whitening deconvolution - compared with well-driven deconvolution
}

\author{
Li Guofa ${ }^{1,2 *}$, Zhou Hui ${ }^{1,2}$ and Zhao Chao ${ }^{1,2}$ \\ ${ }^{1}$ State Key Laboratory of Petroleum Resource and Prospecting, China University of Petroleum, Beijing 102249, China \\ ${ }^{2}$ Key Laboratory of CNPC Geophysical Exploration, China University of Petroleum, Beijing 102249, China
}

\begin{abstract}
Deconvolution is widely used to increase the resolution of seismic data. To compare the resolution ability of conventional spectrum whitening deconvolution to thin layers with that of welldriven deconvolution, a complex sedimentary geological model was designed, and then the simulated seismic data were processed respectively by each of the two methods. The amplitude spectrum of seismic data was almost white after spectrum whitening, but the wavelet resolution was low. The amplitude spectrum after well-driven deconvolution deviated from white spectrum, but the wavelet resolution was high. Further analysis showed that if an actual reflectivity series could not well satisfy the hypothesis of white spectrum, spectrum whitening deconvolution had a potential risk of wavelet distortion, which might lead to a pitfall in high resolution seismic data interpretation. On the other hand, the wavelet after welldriven deconvolution had higher resolution both in the time and frequency domains. It is favorable for high resolution seismic interpretation and reservoir prediction.
\end{abstract}

Key words: Well-driven, high resolution, spectrum whitening, deconvolution, seismic wavelet

\section{Introduction}

Spectrum whitening deconvolution is a widely-used method for increasing the resolution of seismic data (Mou et al, 2007; Jia et al, 2002). It is supposed that the spectrum of a reflectivity series is approximately white. Its objective is to whiten the spectrum of seismic data in an effective frequency band (Li et al, 2001; Chen and Zhou, 2000).

Using the basic idea of well-constrained impedance inversion, the well-driven high resolution seismic data processing method successfully introduces well-constraint before the stage of reservoir inversion to the seismic data processing stage (Kaderali et al, 2007; Spikes et al, 2008). By introducing the reflectivity series from well log data, the deconvolution problem is transformed from statistical to deterministic (Baan, 2008; Wang, 2006). Well-driven deconvolution, which ensures more reliable resolution after deconvolution, evaluates and determines the deconvolution operator by wavelet. Although the application of well-driven deconvolution has not been as popular as spectrum whitening deconvolution, its advantages over spectrum whitening have gradually attracted more attention.

For comparing the two methods, referring to the actual sedimentary structure in the Dagang Oilfield, Bohai Bay Basin, China, a continental sedimentary thin interbedded model was designed, and the simulated seismic data were respectively processed by spectrum whitening deconvolution

*Corresponding author. email: lgfseismic@126.com

Received September 5, 2008 and well-driven deconvolution. The results illustrated the advantages of well-driven deconvolution, and meanwhile revealed the potential risks of spectrum whitening deconvolution.

\section{Spectrum whitening deconvolution and its problems}

The basic idea of spectrum whitening deconvolution is to whiten the amplitude spectrum of seismic data in a specific frequency band. Assuming that the spectrum of a reflectivity series is approximately white, adjusting the amplitude spectrum of seismic record is equivalent to adjusting that of the wavelet (Li et al, 2008).

Spectrum modeling and spectrum modulation techniques are often used to improve the resolution of seismic records in order to control the amplification of noise after spectrum whitening (Rosa and Ulrych, 1991; Zhao et al, 1996). The amplitude spectrum of the wavelet is simulated by smoothing that of the seismic record, and then a spectrum whitening operator is estimated in the frequency domain. In fact, these methods can be treated as spectrum whitening deconvolution in the frequency domain, and they also need the same basic hypotheses as the time domain methods (Baan and Pham, 2008; Velis, 2008).

The two amplitude spectra shown in Fig. 1 are quite different and it is difficult to simulate one by the other. In fact, Fig. 1(a) is the amplitude spectrum of a $50 \mathrm{~Hz}$ Ricker wavelet, and Fig. 1(b) is the amplitude spectrum of a seismic record which is generated by convoluting the Ricker wavelet with four $20 \mathrm{~ms}$ spaced reflection coefficients with equal 
amplitude. It is seen that if the reflectivity series is not a white spectrum, it is difficult to simulate the amplitude spectrum of wavelet from that of seismic record. Analysis of actual well $\log$ data indicates that the spectrum of a reflectivity series is close to the blue spectrum instead of the expected white

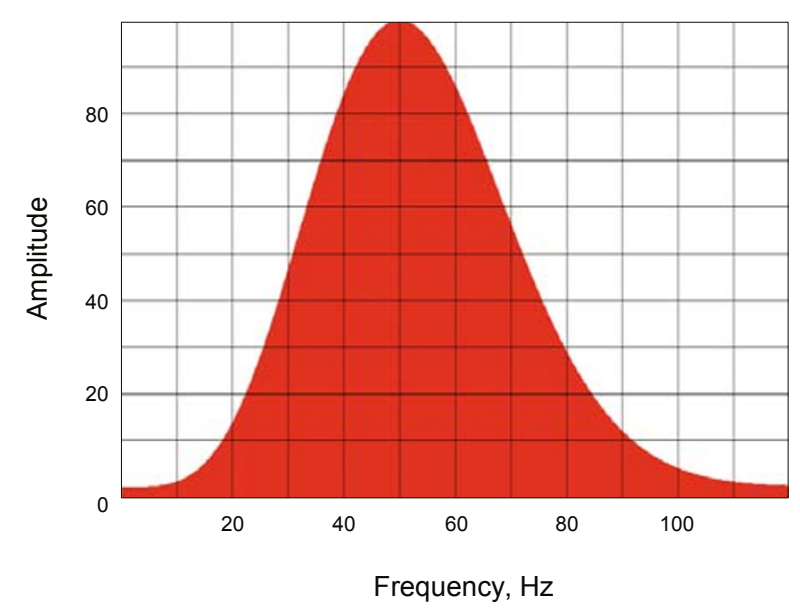

(a) one (Walden and Hosken, 1985). In object-oriented seismic data processing, the time window for spectrum whitening is generally short around the target reflection. As a result, the spectrum of a reflectivity series in the window is far from white spectrum due to inadequacy of the statistics.

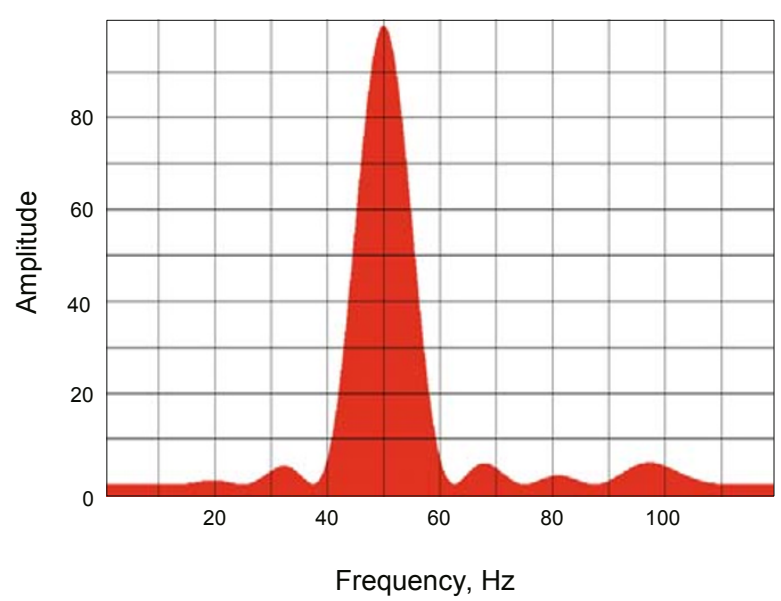

(b)

Fig. 1 Amplitude spectrum of a $50 \mathrm{~Hz}$ Ricker wavelet (a) and the amplitude spectrum of a seismic record generated by convoluting the wavelet with four $20 \mathrm{~ms}$ spaced reflection coefficients with equal amplitude (b)

Another problem of spectrum whitening deconvolution is the neglect of phase effects on wavelet resolution. The wavelet resolution can be most reliably evaluated on the basis of its waveform. If it is evaluated in the frequency domain, amplitude and phase spectra of wavelet should be taken into account together. Now the spectrum whitening deconvolution

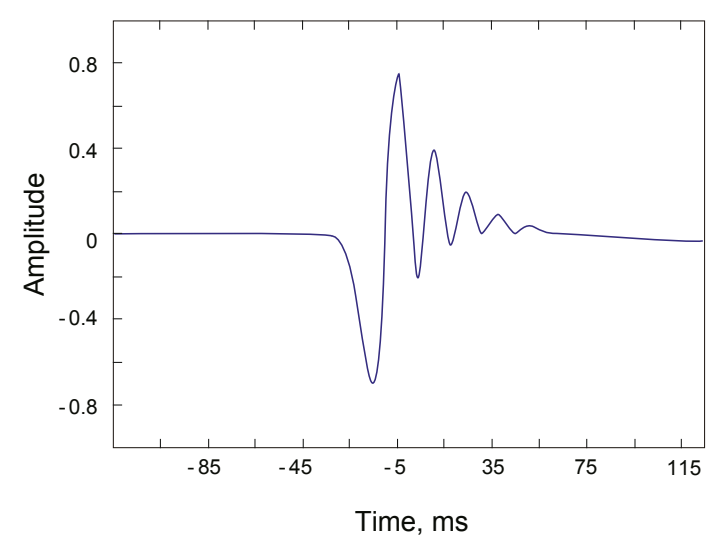

is applied to a $30 \mathrm{~Hz}$ Ricker wavelet, and its phase spectrum is changed. Fig. 2 is the waveform and its amplitude spectrum after the deconvolution. It is seen that the amplitude spectrum is whitened, but the wavelet has more side lobes since the wavelet is no longer zero-phase. Spectrum whitening does not substantially increase the resolution of seismic wavelet.

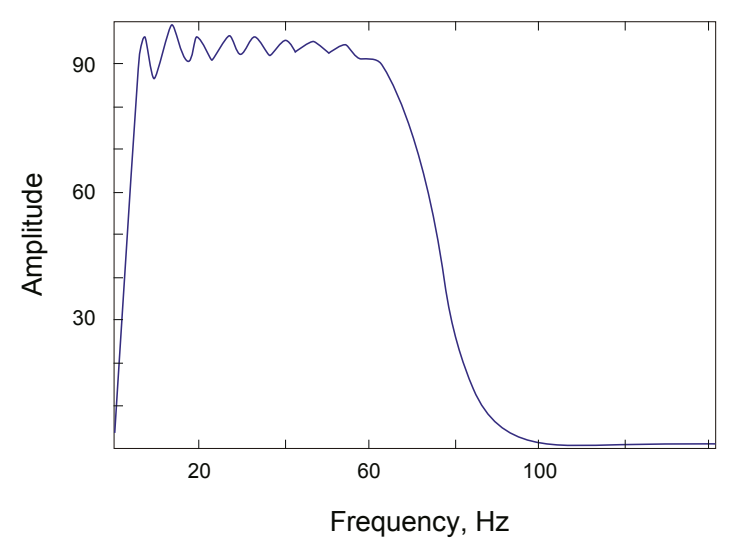

Fig. 2 Waveform (left) and amplitude spectrum (right) of the Ricker wavelet after the spectrum whitening deconvolution and phase alteration

\section{Basic principle of well-driven deconvolution}

The reason why spectrum whitening deconvolution needs the hypothesis of a white spectrum is that, when the reflectivity series is unknown, the wavelet can not be accurately estimated from the seismic record. However, if the well $\log$ data near seismic traces contain velocity and density information, reflectivity coefficients can be calculated from the well log data. Therefore, the wavelet can be estimated directly from the seismic record without the basic hypothesis above mentioned. It is convenient to improve the resolution with the known wavelet (Li et al, 2005). 
Supposing velocity and density from well log data to be $v(z)$ and $\rho(z)$, a seismic trace near the well to be $x(t)$, the basic procedure of well-driven deconvolution is as follows:

1) Reflectivity coefficients $\xi^{\prime}(z)$ are calculated from $v(z)$ and $\rho(z)$ by:

$$
\xi^{\prime}(z)=\frac{\rho(z) v(z)-\rho(z-\Delta z) v(z-\Delta z)}{\rho(z) v(z)+\rho(z-\Delta z) v(z-\Delta z)}
$$

2) The reflectivity coefficients $\xi^{\prime}(z)$ are transferred to the time domain from the depth domain using velocity $v(z)$, and is denoted as $\xi^{\prime}(t)$;

3) The reflectivity coefficients $\xi(t)$, which agree with the well-nearby seismic trace in time-depth relationship, are generated by means of well-ties;

4) A time window $\left[T_{1}, T_{2}\right]$, within which the well-nearby seismic trace agrees well with the synthetic trace, is selected, and wavelet $w(t)$ is estimated in this window:

$$
w(t)=x(t) * \xi^{-1}(t)
$$

5) Based on the signal to noise ratio and effective frequency band of seismic data, an expected output zerophase wavelet $b(t)$ is defined;

6) From the estimated wavelet $w(t)$ and the expected wavelet $b(t)$, a deterministic deconvolution operator $a(t)$ is calculated:

$$
a(t)=b(t) * w^{-1}(t)
$$

7) Seismic trace $x(t)$ is convoluted with the operator $a(t)$ to output the resolution-improved seismic record $y(t)$ :

$$
y(t)=x(t) * a(t)
$$

From the above procedure it can be seen that the key to well-driven deconvolution is the estimation of wavelet. The following notices should also be taken in practical applications.

1) Synthetic trace and well-nearby seismic trace should have the same time-depth relationship, so fine well-ties are vital for wavelet estimation.

2) Well-nearby seismic traces should have good signal to noise ratio and imaging quality. Otherwise, the wavelet should be estimated by observing the similarity of synthetic and well-nearby traces, and should not be directly calculated from Eq. (3).

3) The length of the time window $\left[T_{1}, T_{2}\right]$ should be at least three times the wavelet duration for stable estimation.

\section{Analysis and comparison on the basis of synthetic data}

To make clear the potential problems of spectrum whitening deconvolution and to verify the ability of welldriven deconvolution in high-resolution seismic data processing, a continental sedimentary thin interbedded model is designed on the basis of geological information from the Dagang Oilfield. The simulated seismic data are respectively processed by spectrum whitening and welldriven deconvolution.

Fig. 3 shows the continental sedimentary thin interbedded model in the time-space domain. The velocities of sand and shale are $3000 \mathrm{~m} / \mathrm{s}$ and $2800 \mathrm{~m} / \mathrm{s}$ respectively, and the greatest thickness of sand bodies is $20 \mathrm{~m}$. The wave fields are simulated by wave equations and are processed by pre-stack time migration. Fig. 4 is the seismic section after migration in which two pseudo-logs are inserted. It is noticed that some sand bodies in Fig. 3 are not well resolved in Fig. 4 because of wavelet interference.

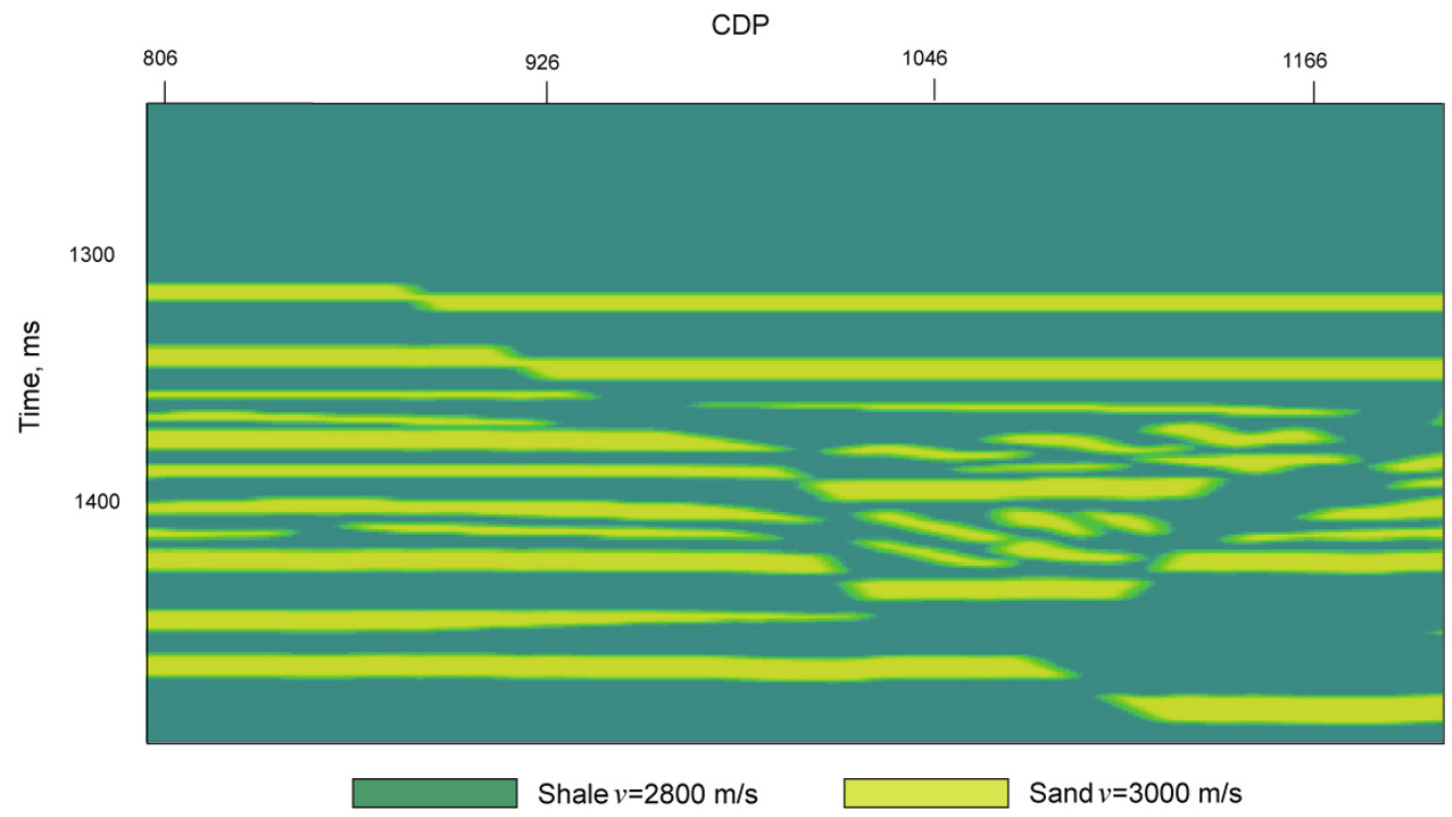

Fig. 3 A continental sedimentary sand and shale thin interbedded model 


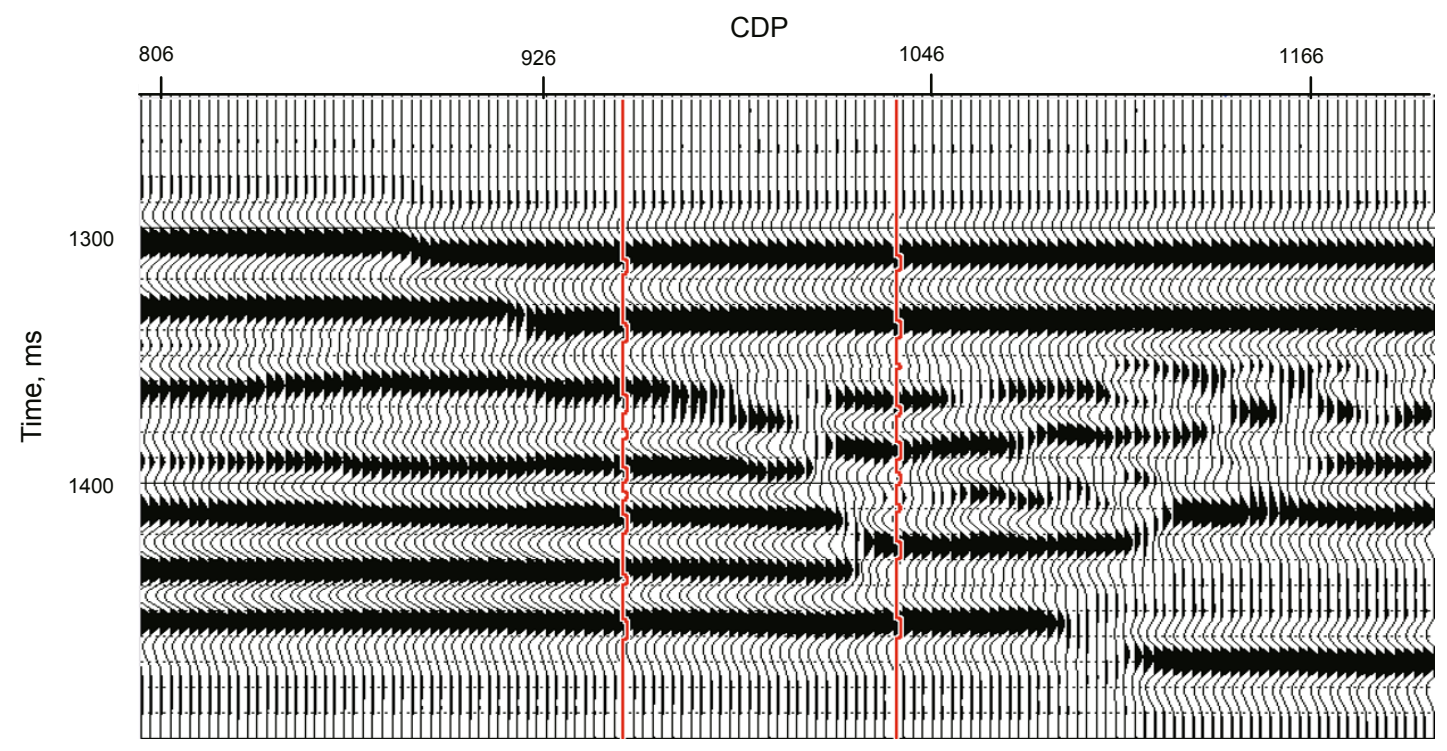

Fig. 4 Seismic section after pre-stack time migration

Spectrum whitening deconvolution is first used to increase the resolution of Fig. 4. The result is shown in Fig. 5. Compared with Fig. 4, the resolution is improved to some extent. The amplitude spectrum after the deconvolution is shown in Fig. 6. It is seen that the energy of each frequency component below $85 \mathrm{~Hz}$ is almost uniform. Spectrum whitening result is satisfactory if it is evaluated by amplitude spectrum.

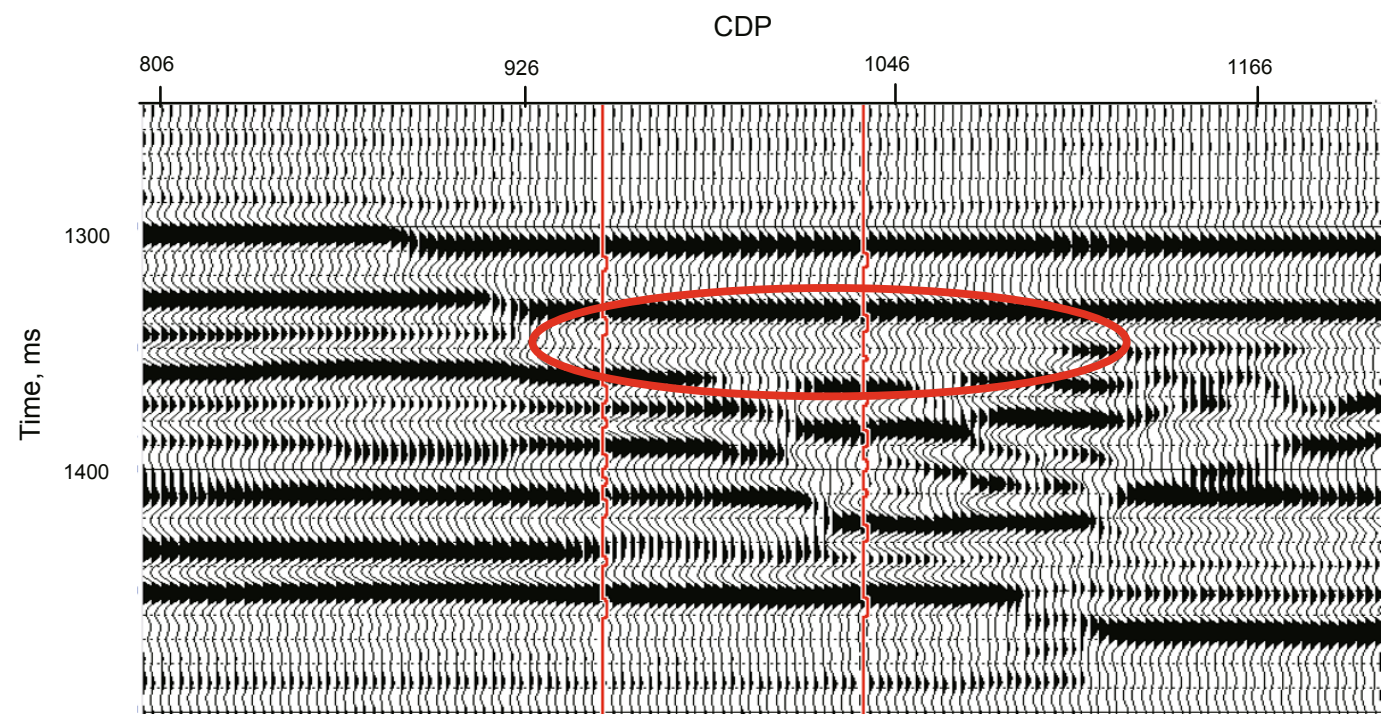

Fig. 5 Seismic section after spectrum whitening deconvolution

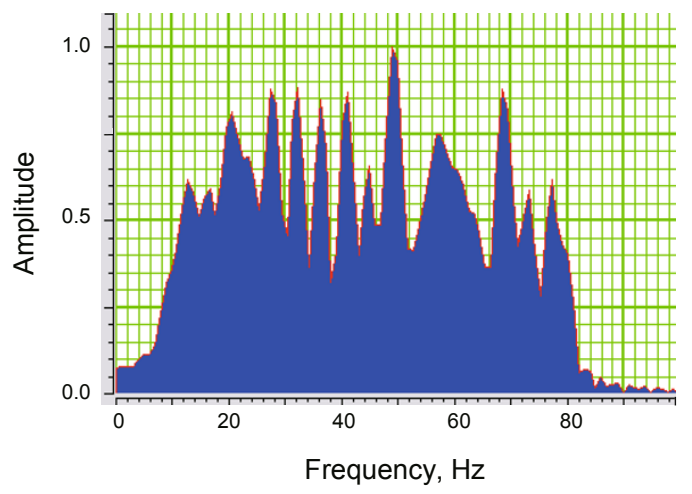

Fig. 6 Amplitude spectrum of seismic data after spectrum whitening deconvolution
The resolution can only be approximately evaluated by using the amplitude spectrum of seismic data. Strictly speaking, it should be evaluated by the waveform and spectrum of the wavelet. Therefore, the seismic wavelet after spectrum whitening deconvolution is extracted by using the well logs shown in Fig. 5. The extracted wavelet and its spectrum are shown in Figs. 7(a) and 7(b). It is seen from Fig. 7(a) that wavelet shape is not satisfactory since the main lobe is wide and the side lobes are strong. From Fig. 7(b) it is noticed that the amplitude under $32 \mathrm{~Hz}$ is strong, the amplitude above $32 \mathrm{~Hz}$ is weak, and the amplitude around $40 \mathrm{~Hz}$ is only half of the peak. As a result, the resolution after spectrum whitening deconvolution is not satisfactory if evaluated by wavelet. 


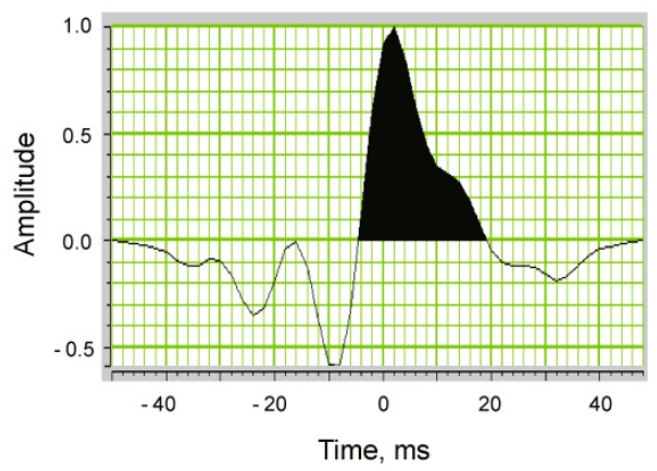

(a)

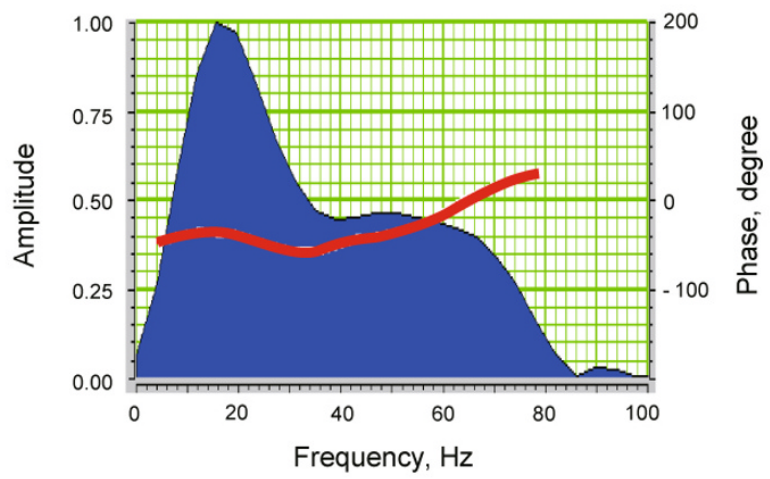

(b)

Fig. 7 Wavelet (a) and its spectrum (b) after spectrum whitening deconvolution

Next, well-driven deconvolution is applied to the synthetic data shown in Fig. 4, and the result is shown in Fig. 8. By comparing Fig. 8 with Fig. 5, it is seen that the circled thin layer in Fig. 8 is well resolved, but it does not appear in Fig. 5 after spectrum whitening. From the comparison of seismic data, it is known that the resolution after well-driven deconvolution is higher than that after spectrum whitening. Fig. 9 is the amplitude spectrum of seismic data after well-driven deconvolution. The spectrum in the effective band is not well whitened compared to that after spectrum whitening deconvolution shown in Fig. 6. Consequently, if it is evaluated by the amplitude spectrum of seismic data, it seems that the resolution after spectrum whitening is higher than that after well-driven deconvolution. It is in conflict with the conclusion drawn from the comparison of seismic sections.

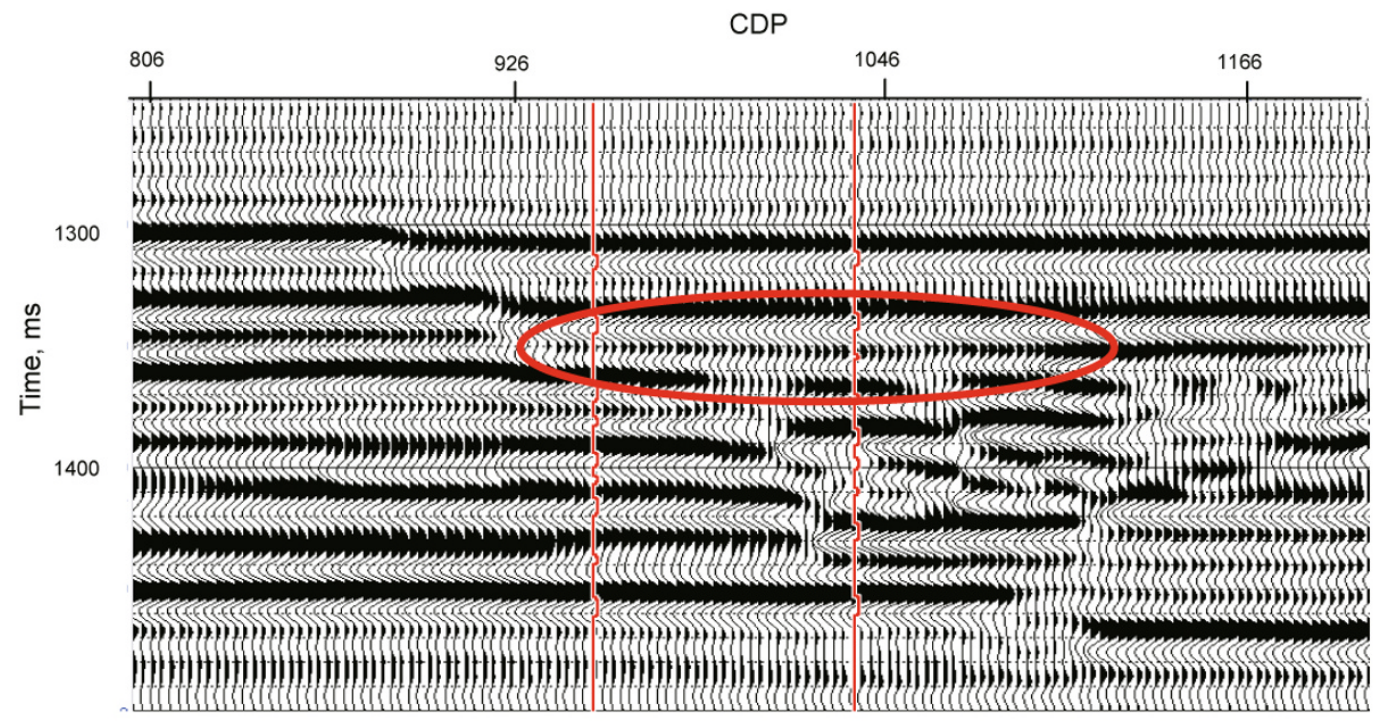

Fig. 8 Seismic section after well-driven deconvolution

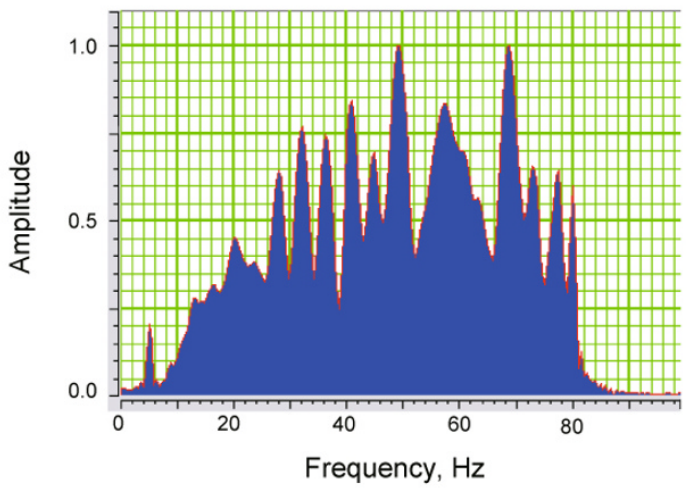

Fig. 9 Amplitude spectrum of seismic data after well-driven deconvolution
In order to clarify the contradiction, the resolution after well-driven deconvolution is further evaluated by the wavelet. Fig. 10 shows the extracted wavelet and its amplitude spectrum after well-driven deconvolution. Compared with the wavelet after spectrum whitening shown in Fig. 7(a), the wavelet after well-driven deconvolution shown in Fig. 10(a) has concentrated energy, weak side lobes and symmetric waveform in the time domain. It also can be seen from amplitude spectrum of wavelet shown in Fig. 10(b) that in the frequency domain the amplitude of each frequency within the effective band is almost uniform. Therefore, if it is evaluated by wavelet, the well-driven deconvolution can improve resolution better than spectrum whitening. 


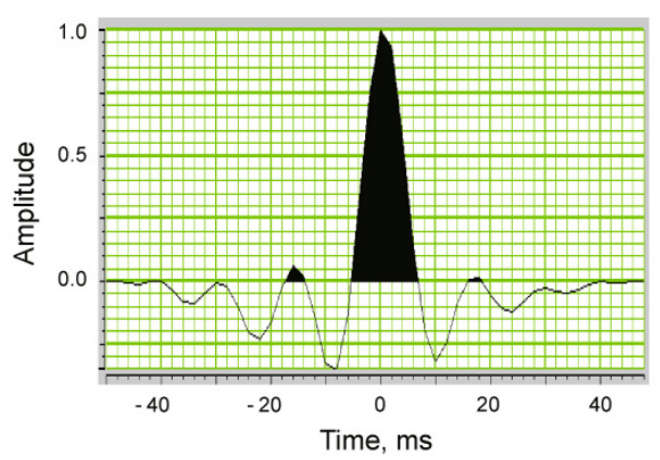

(a)

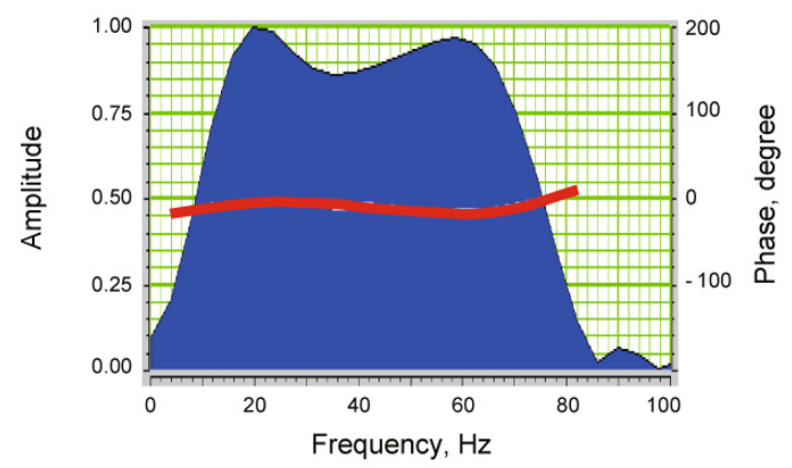

(b)

Fig. 10 Wavelet (a) and its spectrum (b) after well-driven deconvolution

From the above experiments and analysis, it can be seen that, if evaluated by the spectrum of seismic record itself, the seismic data after spectrum whitening seem to have higher resolution since the spectrum is more close to white. However, if it is evaluated by wavelet, the actual resolution is not satisfactory. The seismic data after well-driven deconvolution seem to have lower resolution since the spectrum deviates from white. However, if it is evaluated by wavelet, the actual resolution after well-driven deconvolution is higher than that after spectrum whitening since the waveform and spectrum of wavelet is close to the expected demand. The following is the analysis to explain why this conflicting phenomenon occurs.

Fig. 11 is the amplitude spectrum of a reflectivity series calculated from the well log. It can be obviously seen that the amplitude spectrum is far from white as supposed in the spectrum whitening method. The whole trend of the spectrum is that at low frequencies the amplitudes are weak, and at high frequencies they are strong. This is similar to that after well-driven deconvolution. As a result, the conclusion can be drawn that, if the spectrum of the actual reflectivity series deviates from white, the spectrum whitening deconvolution still tries to alter the spectrum of seismic record to white at the expense of making the spectrum of the wavelet away from white. Consequently, the resolution of seismic data seems to be enhanced, but the actual reflectivity series of subsurface structure is not actually recovered.

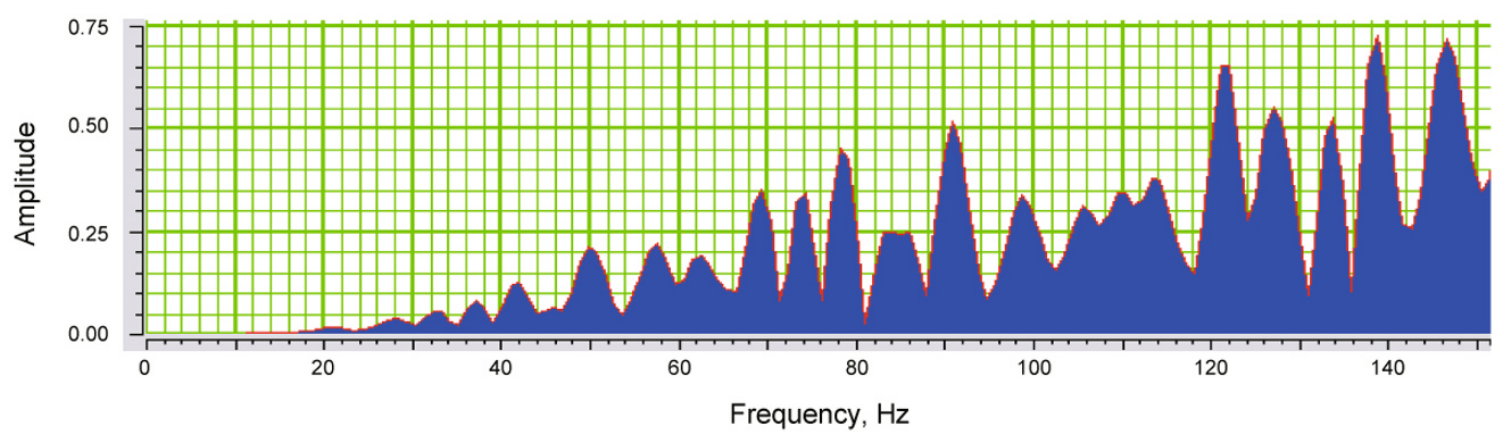

Fig. 11 Amplitude spectrum of a reflectivity series calculated from well log

\section{Conclusions}

Spectrum whitening deconvolution is a widely-used method for improving seismic resolution. Although we have known that the reflectivity series of actual subsurface structures does not satisfy the hypothesis of white spectrum, the experiments and analysis about the hypothesis have not been systematically investigated, such as the negative effects on seismic resolution and pitfalls that may occur from the hypothesis.

The experiments in this paper demonstrated that, if the spectrum of an actual reflectivity series deviates from white, spectrum whitening deconvolution tried to modulate the spectrum of seismic data to the white spectrum at the expense of making the spectrum of the wavelet deviate from white.
In this way, the spectrum of seismic data after spectrum whitening seemed to be white, but the reflectivity series of the subsurface structure was not actually recovered since the waveform and spectrum of the wavelet were distorted.

By means of introducing reflectivity series from well logs, well-driven deconvolution avoided the basic hypothesis in spectrum whitening deconvolution. The wavelet after well-driven deconvolution, evaluated by both waveform and spectrum, had higher resolution. It is favorable for high resolution seismic interpretation and reservoir prediction.

\section{Acknowledgements}

This research was financially supported by National 973 Key Basic Research Development Program (N0. 
2007CB209608) and National 863 High Technology Research Development Program (No. 2007AA06Z218).

\section{References}

Baan M V. Time-varying wavelet estimation and deconvolution by kurtosis maximization. Geophysics. 2008. 73(2): 11-18

Baan M V and Pham D T. Robust wavelet estimation and blind deconvolution of noisy surface seismics. Geophysics. 2008. 73(5): $37-46$

Chen C R and Zhou X X. Improving resolution of seismic data using wavelet spectrum whitening. Oil Geophysical Prospecting. 2000. 35(6): 703-709 (in Chinese)

Jia L H, Wu C J, Luo Y X, et al. High resolution seismic data processing techniques. Geophysical Prospecting for Petroleum. 2002. 41(4): 484-488 (in Chinese)

Kaderali A, Jones M and Howlett J. White Rose seismic with well data constraints: A case history. The Leading Edge. 2007. 26(6): 742-754

Li G F, Mou Y G and Wang P. A interactive technique for seismic wavelet extraction. Journal of China University of Petroleum (Edition of Natural Science). 2005. 29(5): 33-36 (in Chinese)

Li G F, Mou Y G, Liu Y Q, et al. Spectrum-modulated technique. Oil
Geophysical Prospecting. 2001. 36(5): 597-601 (in Chinese)

Li G F, Xiong J L, Zhou H, et al. Seismic reflection characteristics of fluvial sand and shale interbedded layers. Applied Geophysics. 2008. 5(3): 219-229

Mou Y G, Chen X H, Li G F, et al. Seismic Data Processing. Beijing: Petroleum Industry Press. 2007. 70-75 (in Chinese)

Rosa A L R and Ulrych T J. Processing via spectral modeling. Geophysics. 1991. 56(8): 1244-1251

Spikes K, Dvorkin J and Schneider M. From seismic traces to reservoir properties: Physics-driven inversion. The Leading Edge. 2008. 27(4): 456-461

Velis D R. Stochastic sparse-spike deconvolution. Geophysics. 2008. 73(1): $1-9$

Walden A T and Hosken J W J. An investigation of the spectral properties of primary reflection coefficients. Geophysical Prospecting. 1985. 33(3): 400-435

Wang Y H. Inverse Q-filter for seismic resolution enhancement. Geophysics. 2006. 71(3): 51-60

Zhao B, Yu S P, Nie X B, et al. Spectral-modeled deconvolution and its application. Oil Geophysical Prospecting. 1996. 31(1): 101-113 (in Chinese)

(Edited by Hao Jie) 